\title{
Bugan Rongjin decoction alleviates inflammation and oxidative stress to treat the postmenopausal knee osteoarthritis through Wnt signaling pathway
}

\author{
Sheng Wang ${ }^{1 \dagger}$, Pei Ding ${ }^{2 \dagger}$, Xiaopeng Xia ${ }^{1}$, Xuexian Chen ${ }^{1}$, Daguo $\mathrm{Mi}^{1}$, Shuijie Sheng ${ }^{3}$, Fulong Gu${ }^{1}$, \\ Zhongwei $\mathrm{Li}^{1}$, Kelei Su${ }^{4^{*}}$ and Yuwei $\mathrm{Li}^{5^{*}}$ (D)
}

\section{${ }^{*}$ Correspondence:} sukelei@163.com;

liyuweiyw@163.com

†Sheng Wang and Pei Ding

contributed equally

${ }^{4}$ Department of Respiratory

Medicine, Affiliated Hospital

of Integrated Traditional

Chinese and Western

Medicine, Nanjing University

of Chinese Medicine,

Number 100, Cross Street,

Hongshan Road, Qixia

District, Nanjing 210028,

Jiangsu, China

${ }^{5}$ Department of Orthopedics and Traumatology, Suzhou

TCM Hospital Affiliated

to Nanjing University

of Chinese Medicine

Number 18 Yangsu Road,

Suzhou 215000, Jiangsu,

China

Full list of author information

is available at the end of the article

\begin{abstract}
Background: Traditional Chinese medicine has been found effective for the therapy of knee osteoarthritis (KOA). This study was aimed at investigating the underlying mechanism of Bugan Rongjin decoction (BGRJ) in treating the postmenopausal KOA.

Results: Ovariectomized rat model of KOA and LPS-induced chondrocytes were successfully constructed for in vivo and in vitro model of postmenopausal KOA. X-ray and hematoxylin-eosin (H\&E) staining showed that BGRJ alleviated pathological damage of articular cartilage in OVX rats with KOA. In addition, BGRJ inhibited inflammation and oxidative stress through decreasing the levels of serum IL-6, IL-1 $\beta$, TNF- $a$ and NO and regulated Wnt signaling pathway by downregulating the expression of Wnt5a and $\beta$-catenin and upregulating the expression of Sox9 and Collagen II in cartilage tissue, detected by immunohistochemistry $(\mathrm{IHC})$ and western blot analysis. Furthermore, Wnt5a silencing reduced the apoptosis of LPS-induced ADTC5 cells, which was further suppressed by the combination of downregulation of Wnt5a and BGRJ.
\end{abstract}

Conclusions: In summary, BGRJ alleviates inflammation and oxidative stress to treat the postmenopausal KOA through Wnt signaling pathway.

Keywords: Bugan Rongjin decoction, Inflammation, Oxidative stress, Wnt signaling pathway, Postmenopausal knee osteoarthritis

\section{Introduction}

Knee osteoarthritis (KOA) is the most common bone degenerative disease in middleaged and elderly people. It is mainly manifested as knee stiffness, pain, swelling, joint deformity, decreased muscle strength of lower limbs and limited activity [1]. Severe cases can affect the patient's daily self-care ability, which is one of the reasons for the elderly's disability [2]. With the accelerating process of population aging, the incidence of KOA is also increasing year by year, which greatly increases the economic burden of society and family [3]. In China, $50 \% \sim 60 \%$ of people over 60 years old suffer from KOA author(s) and the source, provide a link to the Creative Commons licence, and indicate if changes were made. The images or other third party material in this article are included in the article's Creative Commons licence, unless indicated otherwise in a credit line to the material. If material is not included in the article's Creative Commons licence and your intended use is not permitted by statutory regulation or exceeds the permitted use, you will need to obtain permission directly from the copyright holder. To view a copy of this licence, visit http:// creativecommons.org/licenses/by/4.0/. The Creative Commons Public Domain Dedication waiver (http://creativecommons.org/publicdomain/zero/1.0/) applies to the data made available in this article, unless otherwise stated in a credit line to the data. 
[4]. The prevalence of KOA is significantly higher in postmenopausal women than in men of the same age group due to the decrease in estrogen levels in postmenopausal women [5]. The current treatments, such as non-steroidal anti-inflammatory drugs, hyaluronic acid injections, and arthroplasty were demonstrated to be effective, but also accompanied with certain side effects [6]. Furthermore, there is no recommended routine treatment for KOA.

Recently, some promising elements and Chinese herbal compound have been confirmed to slow down the progression of $\mathrm{KOA}$, promote cartilage repair, and alleviate knee joint disorders [7]. KOA belongs to Chinese medicine, "Bi Syndrome", "Bi-bone" areas, which refers to the pain and stiffness or malfunction of the joints [8]. Wind-colddamp-hot impediment causes extremities clonus, knee pain and obstruction of flexed and extended, which leaded to a long time existence of Bi syndrome, further damage to the liver and kidney [9]. Zeng et al. [10] analyzed the rule of medication through data mining and concluded that nourishing liver and kidney, and removing blood stasis and freeing vessel was the main treatment for KOA. Liangchun Zhu, a master of Chinese medicine, has considered that the treatment of KOA can be discussed from the deficiency of liver Yin and loss of nutrient of tendons [11].

Bugan Rongjin decoction (BGRJ) is supplemented and improved basing on the Chen Shiduo's Yangjin Tang. The main ingredients of BGRJ are Fried Radix Paeoniae Alba, Rehmannia, Radix Ophiopogonis, Fried Zizyphus jujube, Morinda officinalis, Angelica sinensis, Papaya, Achyranthes bidentata Blume, Eupolyphaga sinensis Walker and Lumbricus. Paeoniflorin (PF), the main substance of glucosides in Radix Paeoniae Alba, can alleviate the hepatic ischemia/reperfusion (I/R) injury by suppressing inflammation [12]. Catalpol, an iridoid glycoside isolated from the root of Rehmannia, protects against osteoarthritis by inhibiting inflammation, oxidative stress and apoptosis of chondrocytes [13]. Monotropein, an iridoid glycoside isolated from the roots of Morinda officinalis, suppresses the apoptosis and inflammation in chondrocytes, which may be useful for the treatment of osteoarthritis [14]. Angelica sinensis polysaccharide has confirmed to exert antioxidant effect on the oxidative stress in human osteoarthritis [15]. Study has evidenced that Achyranthes bidentata Blume extracts have anti-osteoarthritis and anti-inflammatory effects $[16,17]$. Eupolyphaga sinensis Walker is an ingredient of Huoxuezhitong capsule which has proved to alleviate monosodium iodoacetate-induced osteoarthritis [18]. Therefore, BGRJ is used in the treatment of osteoarthritis.

In this study, we aimed at investigating the underlying mechanism underlying BGRJ in treating the postmenopausal KOA. We constructed the ovariectomized rat model of KOA and cultivated lipopolysaccharide (LPS)-induced chondrocytes to explore the therapeutic effects of BGRJ through regulating inflammatory cytokines and Wnt signaling pathway, which may be potential candidates for the therapy and cure of human postmenopausal KOA.

\section{Results}

\section{BGRJ improves KOA in OVX rats}

OVA modeling process of rats is shown as Fig. 1A and KOA modeling process of rats was shown as Fig. 1B. During the experiment, the body weight of rats in each group changed steadily, and each treatment had no obvious effect on the body weight of rats 


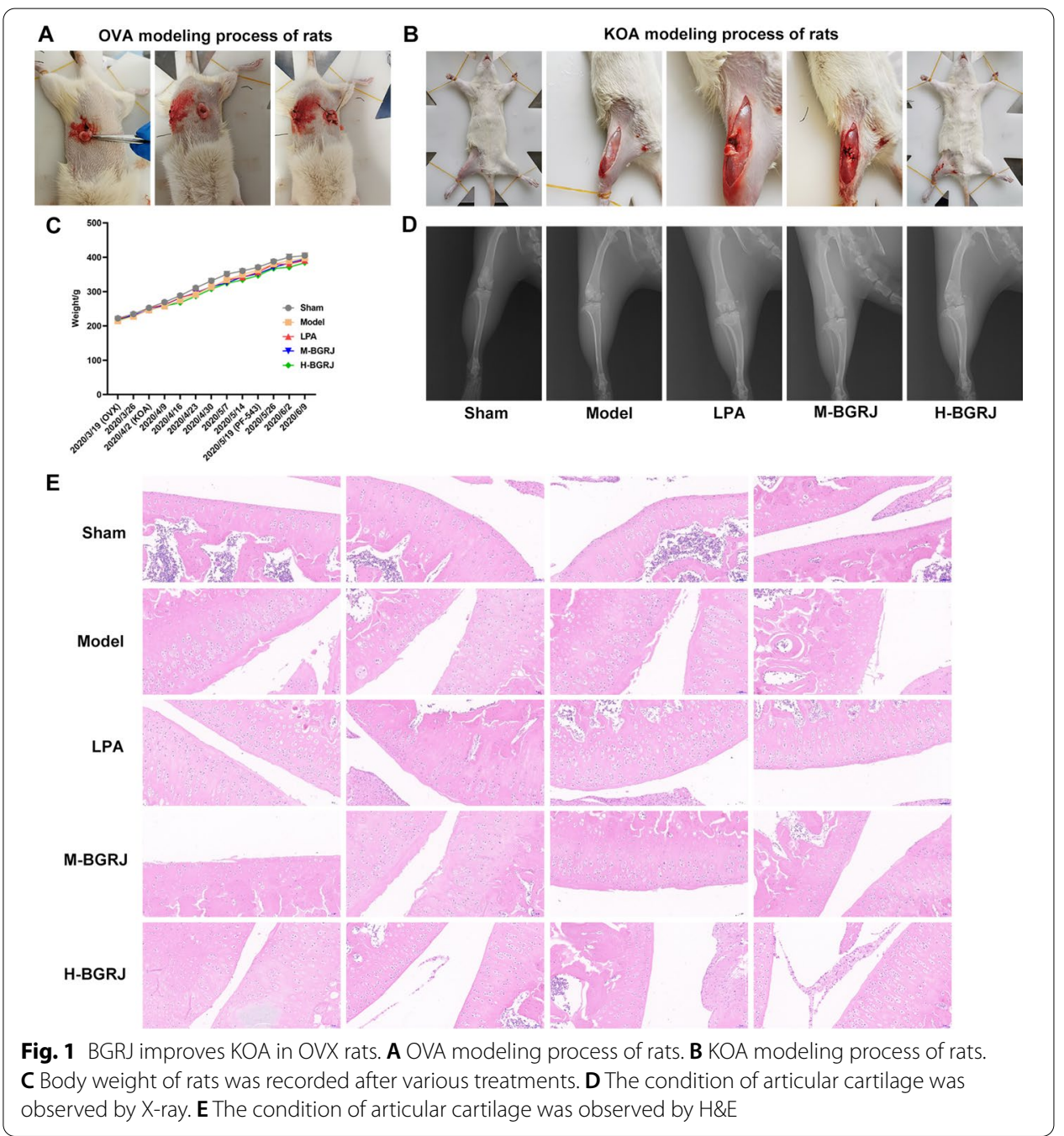

(Fig. 1C). The condition of articular cartilage in these groups was observed by X-ray (Fig. 1D), which was consistent with the results of H\&E (Fig. 1E). The articular cartilage in the Sham group had normal structure, smooth surface without wear and fissure, normal morphology and number of chondrocytes and regular arrangement. In the model group, there were lesions on the surface of articular cartilage, cracks and faults, severe deletion, local reduction and disordered arrangement of chondrocytes. In the LPA group, the articular cartilage was basically flat and the cells were arranged in regular order, but local unsmoothness was still visible. In the M-BGRJ group, the surface of articular cartilage was uneven, with damage and fissures, disordered cell arrangement and reduced cell number. In the H-BGRJ group, the surface of articular cartilage was basically flat, and some coarser cells could be seen locally. The cells were arranged neatly and the morphology was normal.

\section{BGRJ alleviates inflammation and oxidative stress in serum of OVX rats of KOA}

Compared with the sham group, the expression levels of IL-6, IL-1 $\beta$ and TNF- $\alpha$ in serum were all increased in model group. After treatment of BGRJ, the expression levels 
of IL-6, IL-1 $\beta$ and TNF- $\alpha$ in serum were decreased (Fig. 2A-C). Similarly, NO level was also obviously enhanced in model group and was down-regulated by BGRJ treatment (Fig. 2D). Moreover, the high-dose BGRJ has a better alleviating effect on the inflammation and oxidative stress.

\section{BGRJ improves KOA in OVX rats by regulating Wnt signaling pathway}

The expression of Wnt5a (Fig. 3) and $\beta$-catenin (Fig. 4) was increased while Sox9 (Fig. 5) and Collagen II (Fig. 6) was decreased in model group. BGRJ partially reversed the changes of Wnt5a, $\beta$-catenin, Sox 9 and Collagen II in model group. The results of Western blot analysis were also consistent with that of IHC (Fig. 7). Moreover, high-dose BGRJ has better effect than low-dose BGRJ.

\section{Main components of BGRJ confirmed by HPLC}

The main components of BGRJ confirmed by HPLC were nystose, liriopeside, $\beta$-ecdysone, paeoniflorin and verbascoside (Fig. 8A). The contents of nystose, liriopeside, $\beta$-ecdysone, paeoniflorin and verbascoside in the BGRJ were $72,108.3 \mathrm{mg} / \mathrm{L}$, $2294.8 \mathrm{mg} / \mathrm{L}, 10,483.7 \mathrm{mg} / \mathrm{L}, 3722.2 \mathrm{mg} / \mathrm{L}$ and $82.0 \mathrm{mg} / \mathrm{L}$ (Fig. 8B).

\section{Wnt5a expression is decreased in ADTC5 cells after transfection}

The morphology of ADTC5 cells was photographed under microscope (Fig. 9A). After ADTC5 cells were transfected with siRNA-NC and wnt5a siRNA, the transfection effects were confirmed by RT-qPCR analysis (Fig. 9B) and Western blot analysis (Fig. 9C). Compared with CK group, the expression level of wnt5a was not significantly affected by siRNA-NC transfection, and was decreased after ADTC5 cells were, respectively,
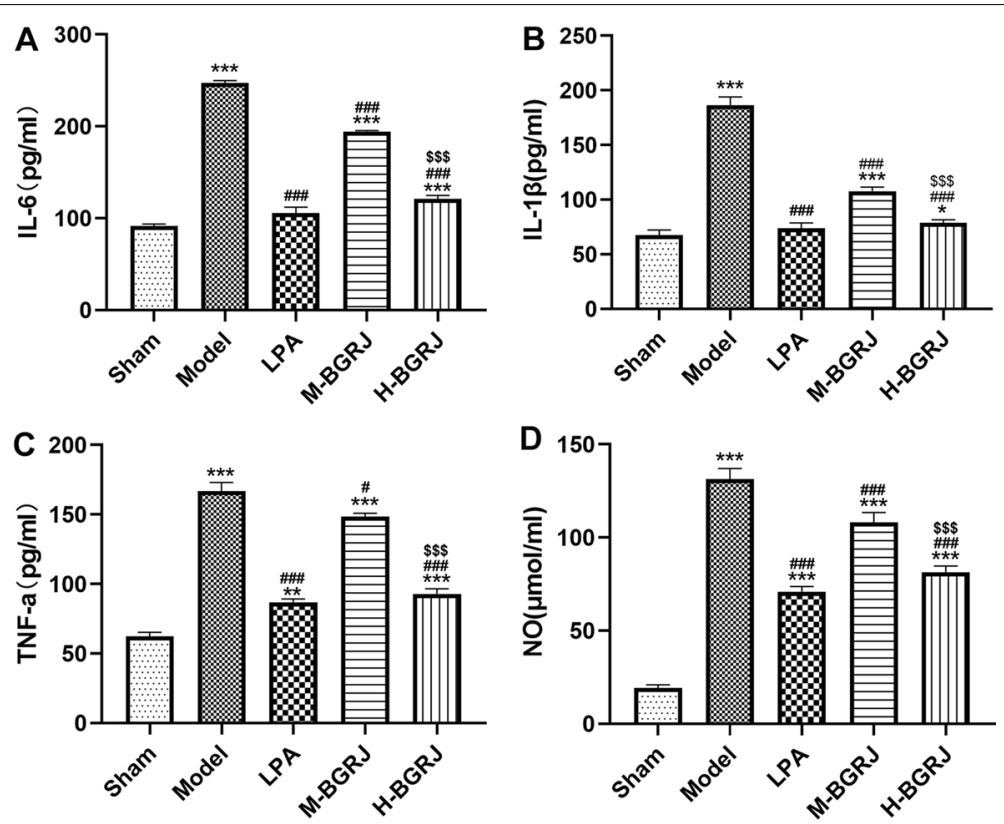

Fig. 2 BGRJ alleviates inflammation and oxidative stress in serum of OVX rats of KOA. The levels of IL-6 (A), $I L-1 \beta(B)$, TNF-a $(\mathbf{C})$ and NO (D) in rat serum were detected by the commercial kits. ${ }^{*} P<0.05,{ }^{* *} P<0.01$ and ${ }^{* * *} P<0.001$ vs. Sham group. ${ }^{\#} P<0.05$ and ${ }^{\# \# \#} P<0.001$ vs. Model group. ${ }^{\$ \$} P<0.001$ vs. M-BGRJ group 


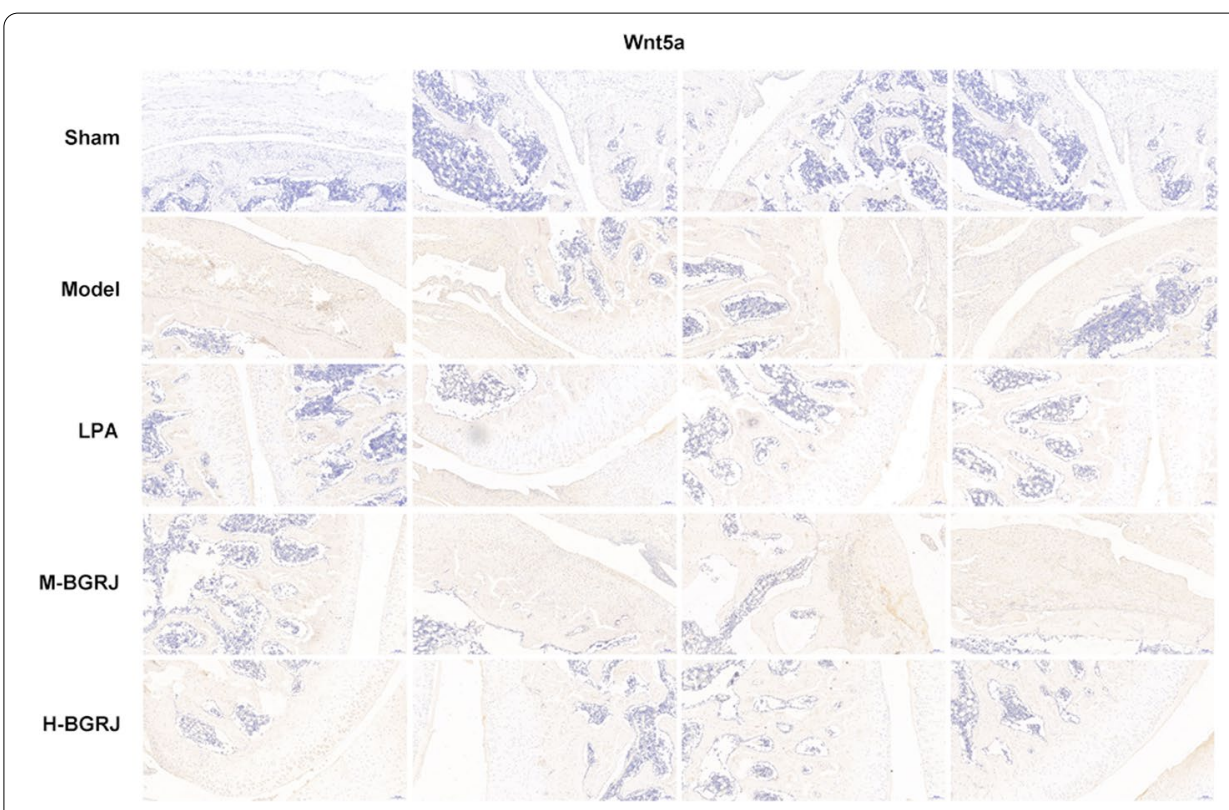

Fig. 3 BGRJ improves KOA in OVX rats by regulating Wnt signaling pathway. Wnt5a expression in articular cartilage was detected by immunohistochemistry

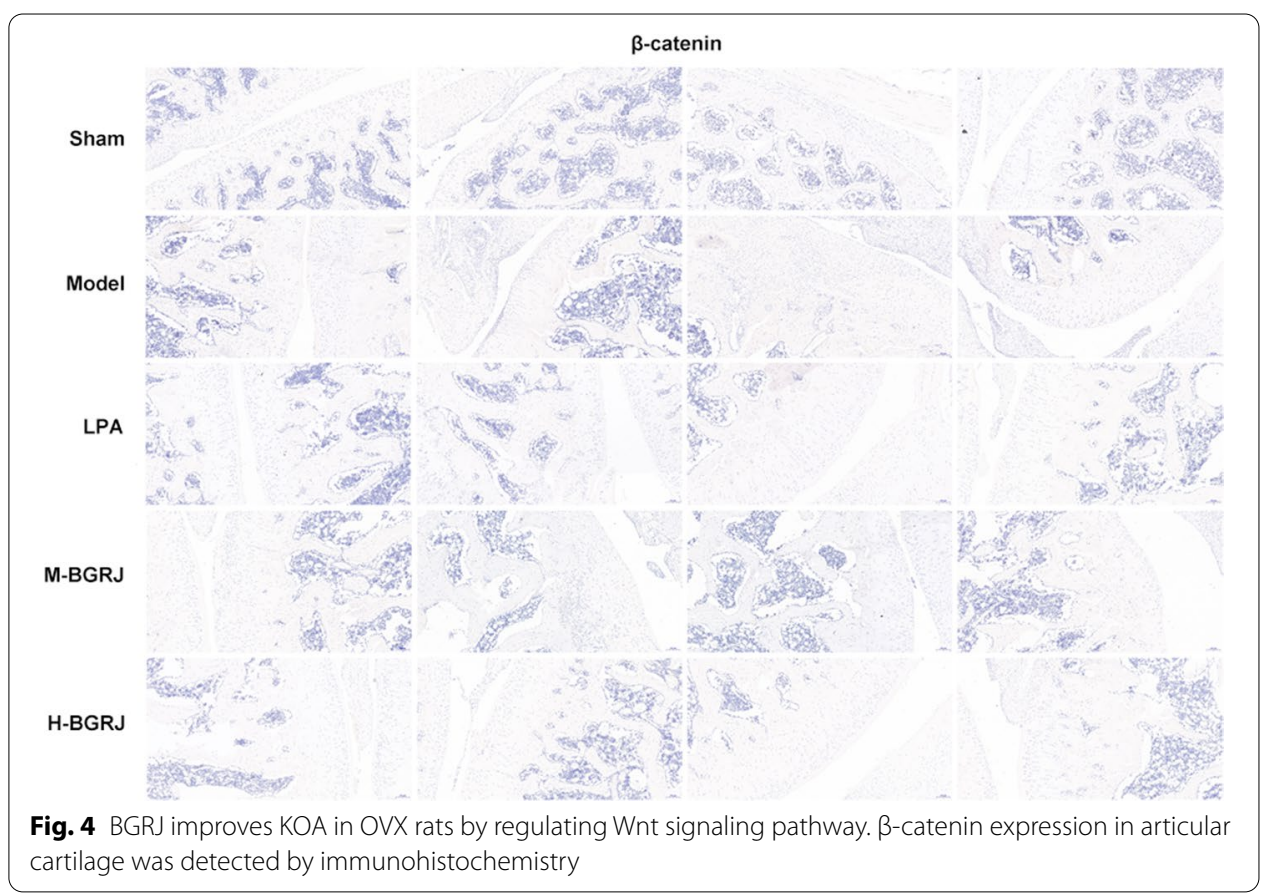

transfected with wnt5a siRNA-1, 2 and 3. We chose wnt5a siRNA-3 for the experiment in the later stage. 


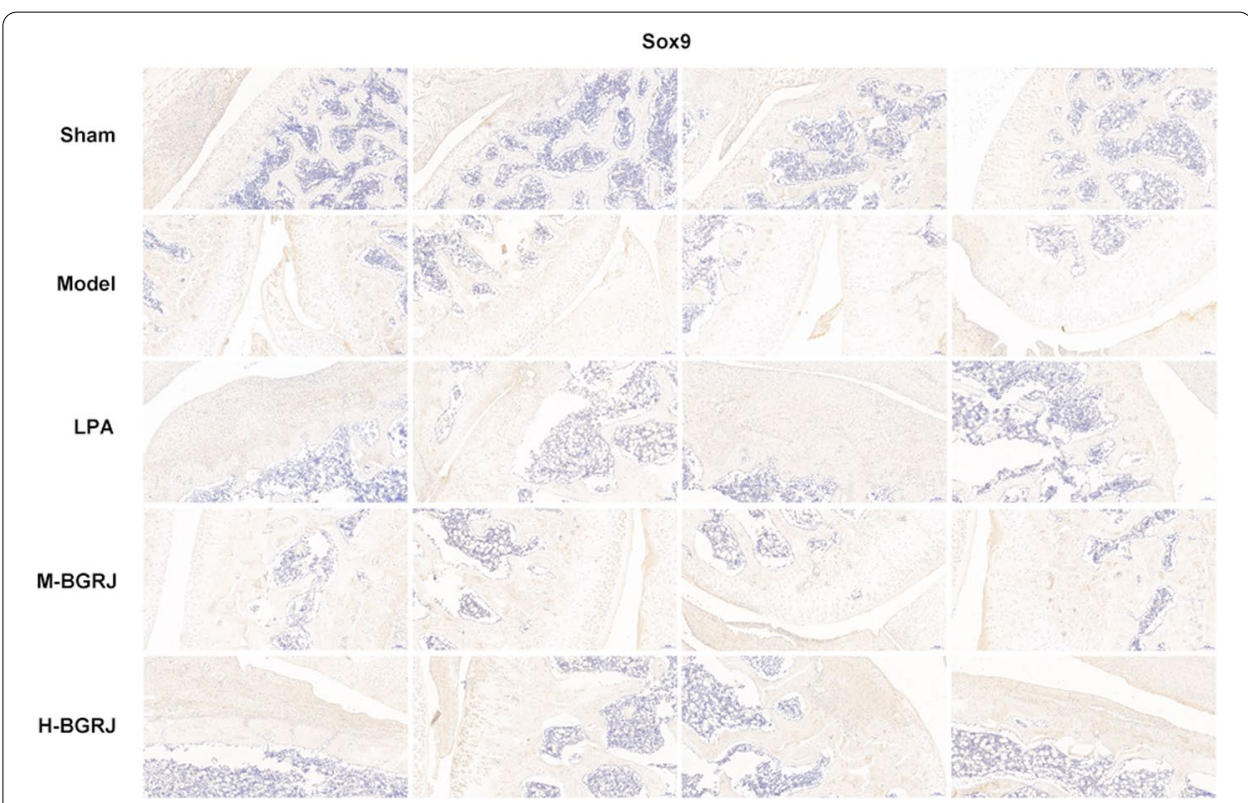

Fig. 5 BGRJ improves KOA in OVX rats by regulating Wnt signaling pathway. Sox9 expression in articular cartilage was detected by immunohistochemistry

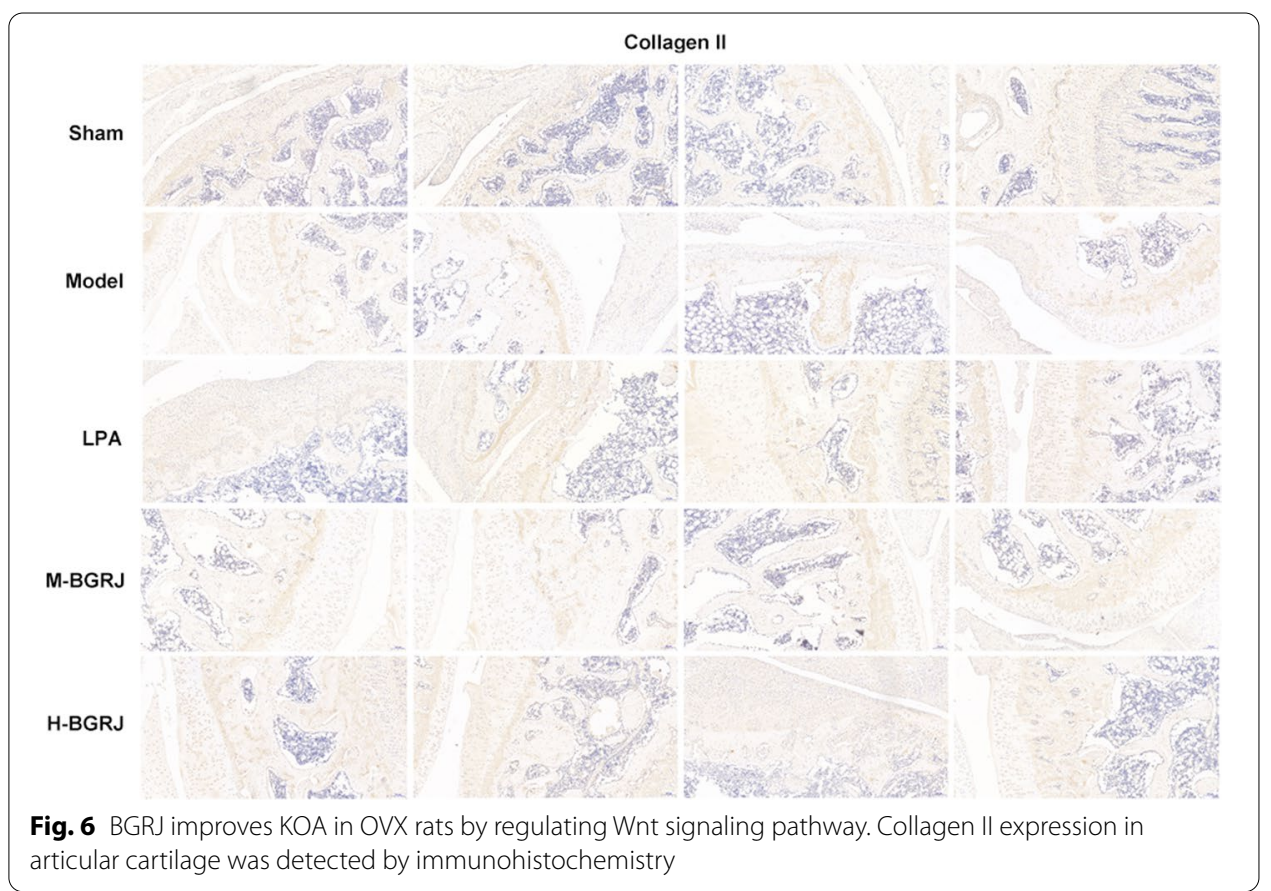

\section{Downregulation of Wnt5a reduces the apoptosis of LPS-induced ADTC5 cells, which is further reduced by BGRJ}

LPS induction obviously promoted the apoptosis of ADTC5 cells while downregulation of Wnt5a could suppress the apoptosis of LPS-induced ADTC5 cells. BGRJ could further reduce cell apoptosis, and the high-dose BGRJ could reduce cell apoptosis better (Fig. 10). 


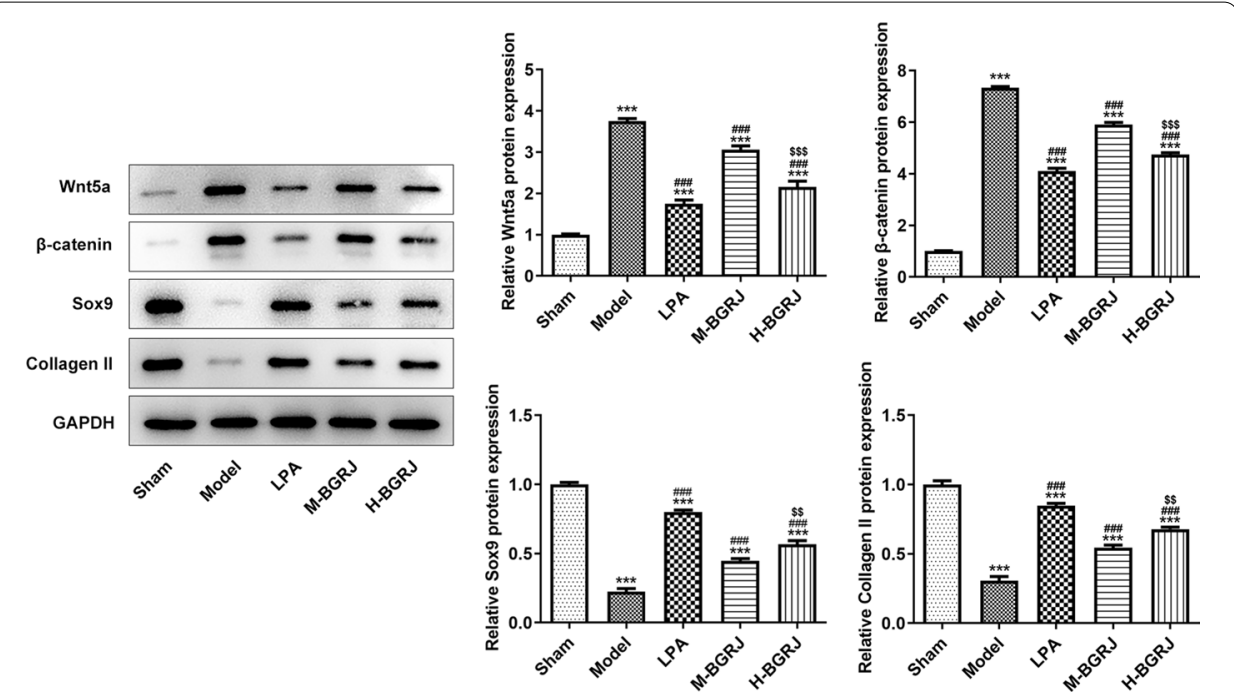

Fig. 7 BGRJ improves KOA in OVX rats by regulating Wnt signaling pathway. The expression of Wnt5a, $\beta$-catenin, Sox9 and Collagen II in articular cartilage was detected by Western blot analysis. ${ }^{* * *} P<0.001$ vs. Sham group. ${ }^{\# \#} P<0.001$ vs. Model group. ${ }^{\$ \$} P<0.01$ and ${ }^{\$ \$ \$} P<0.001$ vs. M-BGRJ group

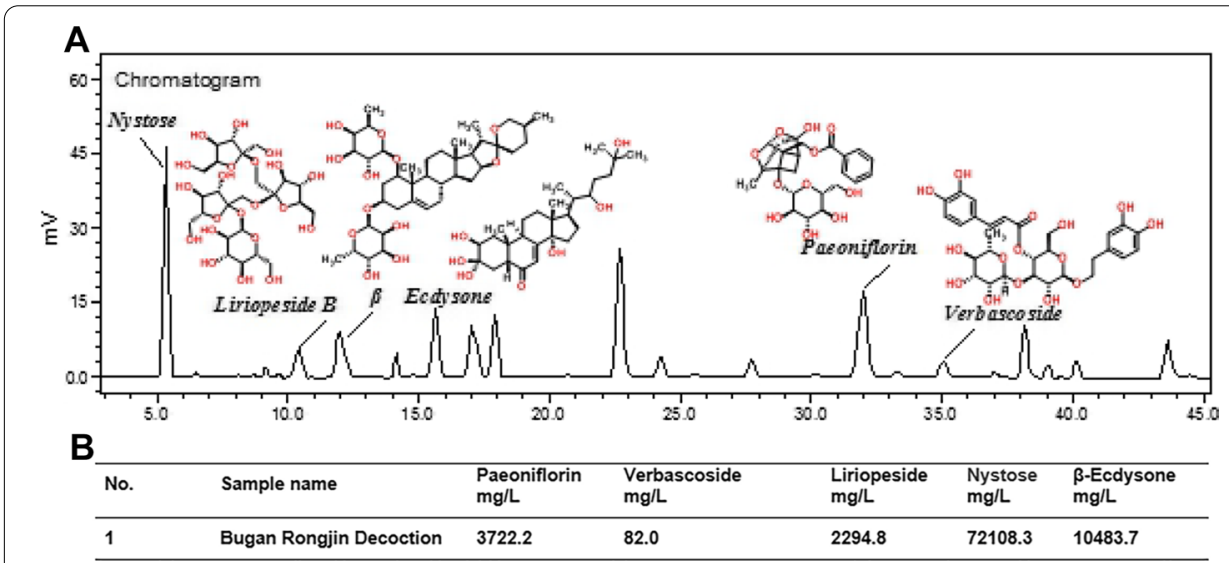

Fig. 8 Main components of BGRJ confirmed by HPLC. A The main components of BGRJ were confirmed by HPLC. B The contents of nystose, liriopeside, $\beta$-ecdysone, paeoniflorin and verbascoside in the BGRJ

\section{Downregulation of Wnt5a regulates Wnt signaling pathway in LPS-induced ADTC5 cells,}

\section{which is further regulated by BGRJ}

LPS induction obviously increased the expression of Wnt5a and $\beta$-catenin and decreased the expression of Sox 9 and Collagen II. Downregulation of Wnt5a inhibited the expression of Wnt5a and $\beta$-catenin and promoted the expression of Sox 9 and Collagen II, which were further regulated by regulated by BGRJ. High-dose BGRJ had better reversing effect on the expression of Wnt5a, $\beta$-catenin, Sox9 and Collagen II in LPS-induced ADTC5 cells (Fig. 11). 

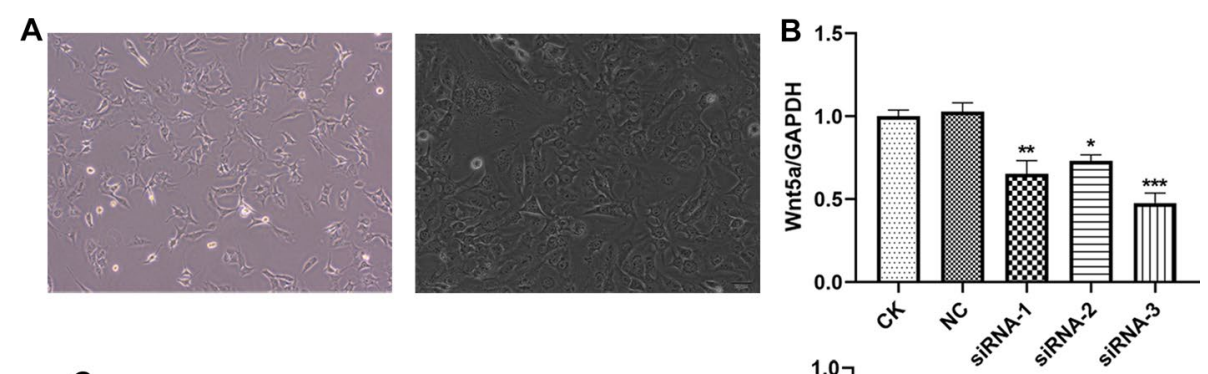

C

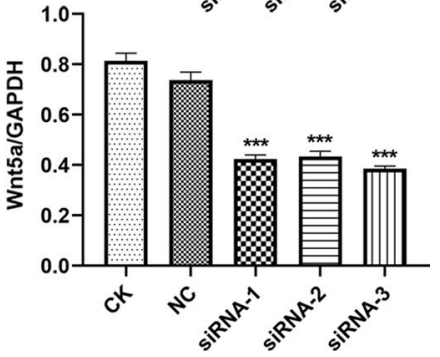

Fig. 9 Wnt5a expression is decreased in ADTC5 cells after transfection. A The morphology of ADTC5 cells were photographed under microscope. B Wnt5a expression in ADTC5 cells after transfection was confirmed by RT-qPCR analysis. C Wnt5a expression in ADTC5 cells after transfection was confirmed by Western blot analysis. ${ }^{*} P<0.05,{ }^{* *} P<0.01$ and ${ }^{* * *} P<0.001$ vs. CK group

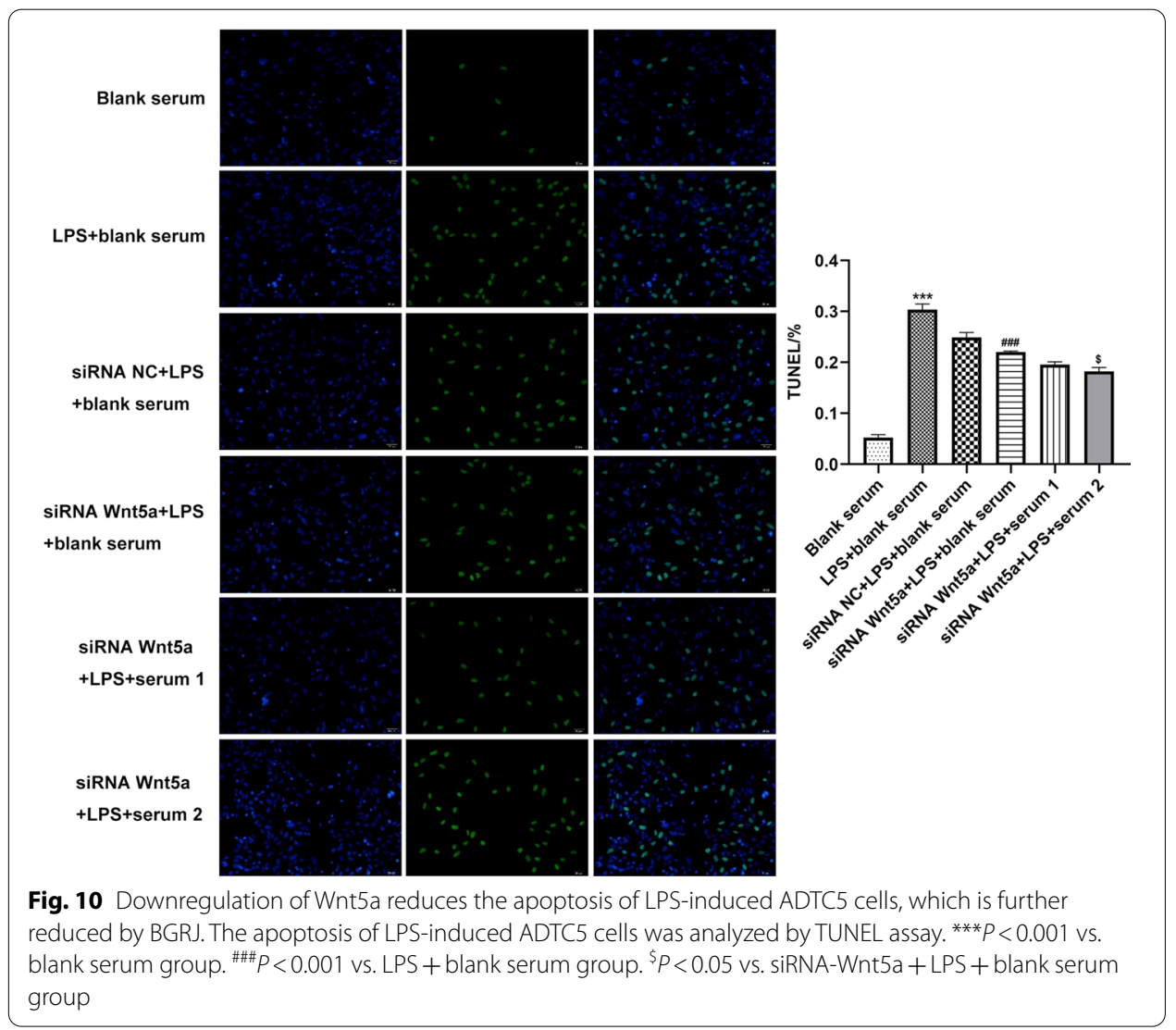




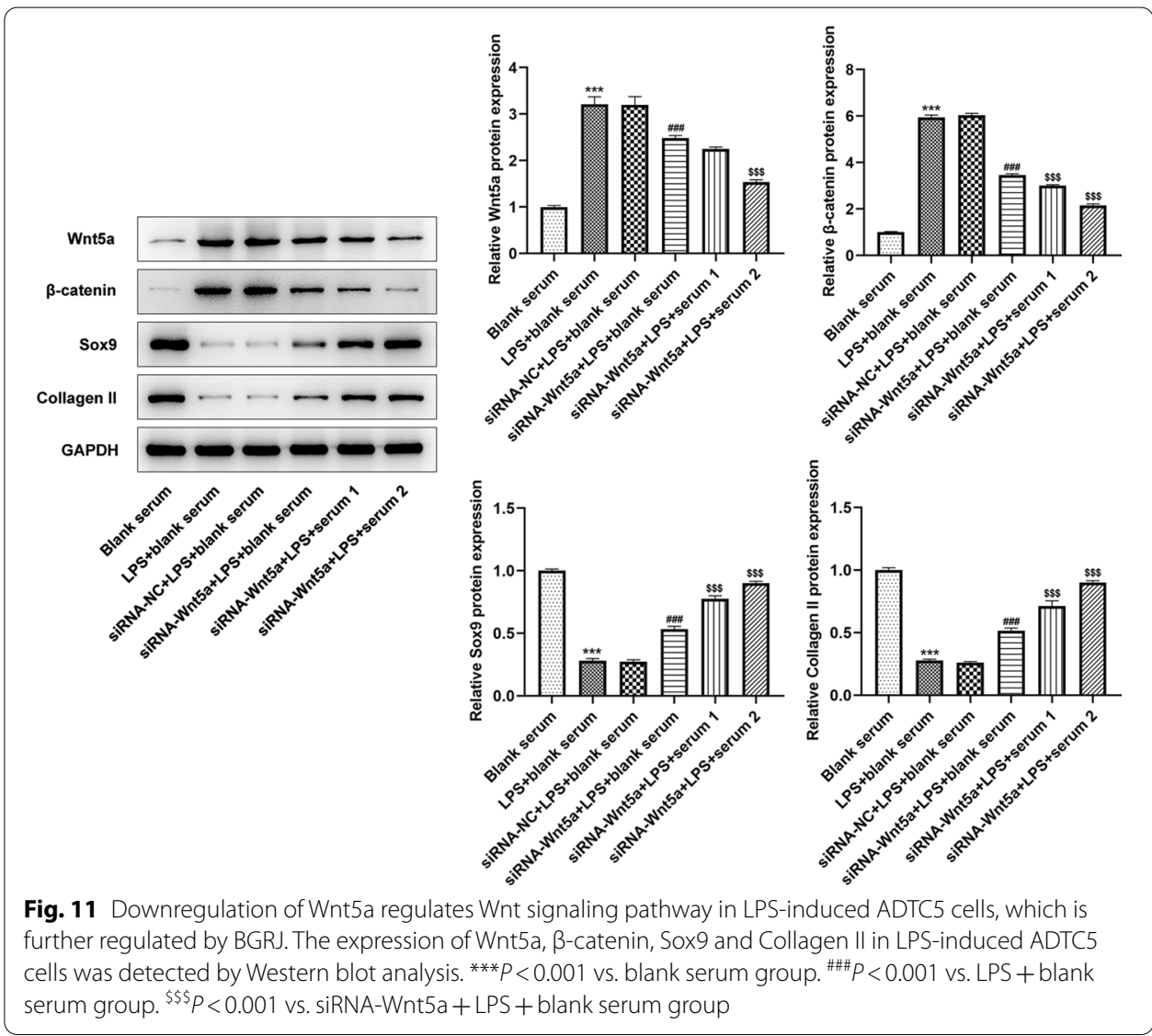

\section{Discussion}

Herbal formulae are complex mixtures of many herbs which contain abundant therapeutic compounds. Therefore, the curative effects of herbal formulae are maximized and the toxicity or adverse effects are minimized by interaction of different herbs [19].

Inflammatory reactions are essential in the progression of KOA, which lead to pain, swelling, and dysfunction and derangement of knee joint [20]. Numerous pro-inflammatory cytokines, chemicals and proteins, such as IL- $1 \beta$, TNF- $\alpha$, MMPs, hyaluronic acid (HA), SOD, NO, and PGE2, were involved in the occurrence of inflammatory reactions $[21,22]$. They not only cause the direct damage to cartilage, but also degrade the extracellular matrix (ECM) and collagen II, which are destroying the knee joint [23]. Herbal therapy significantly regulates the level of many inflammation-related cytokines in the treatment of KOA [24-26]. Here, the levels of IL-6, IL-1 $\beta$, TNF- $\alpha$ and NO were increased in serum of OVX rats of KOA. BGRJ improved the KOA by inhibiting inflammation.

Wnt/ $\beta$-catenin signaling pathway has been demonstrated to regulate the bone remodeling independently or interact with other pathways [27]. The canonical Wnt signaling pathway is over-activated in various OA pathogenesis [28]. Under normal conditions, the $\mathrm{Wnt} / \beta$-catenin signaling pathway is inhibited, and when activated, the overexpression of this pathway promotes the formation of osteoblasts and the release of proteases, during which chondrocytes are degraded, while the block of Wnt signaling induces cartilage formation [29]. The $\mathrm{Wnt} / \beta$-catenin pathway is very important for the expression 
of chondrocyte function. $\beta$-Catenin and its related molecules play an important role in endochondral ossification and participate in chondrocyte differentiation and proliferation [30]. Herbal therapy can suppress the Wnt/ $\beta$-catenin signaling pathway, and alleviate dysfunction of knee joint in KOA patients [31]. Its presence indicated that expression of Wnt5a and $\beta$-catenin was increased and the expression of Sox9 and Collagen II was decreased in OVX rats of KOA, which was partially reversed by BGRJ. In addition, downregulation of Wnt5a could suppress the apoptosis of LPS-induced ADTC5 cells.

The critical pathological characteristic of KOA is that chondrocytes and cartilage matrix were lost which causes degeneration of articular cartilage. Study demonstrated that herbal therapy could suppress the losses of proteoglycans and collagen II and promote the proliferation of chondrocytes [32]. We have found that the apoptosis of ADTC5 cells was increased after LPS induction, which was inhibited by the treatment of BGRJ for LPS-induced ADTC5 cells, indicating the inhibitory effects of BGRJ on inflammation in KOA development.

There are several limitations in the current study. Firstly, we explore the effects of BGRJ in this study at animal and cell levels but not include the clinical samples, and we will verify our hypothesis through clinical trials in the further study. Moreover, because BGRJ is the mixture and we cannot ensure the most important ingredient that exert the effects in BGRJ. Thus, it is needed to perform the separation and purification to explore the main active constituents in BGRJ. Thirdly, how BGRJ acts on Wnt/ $\beta$-catenin signaling was not explored in detail in this study and we will investigate the specific mechanism of BGRJ involved in Wnt/ß-catenin pathway in next study.

\section{Conclusion}

In conclusion, we demonstrated for the first time that BGRJ alleviates inflammation and oxidative stress in treating the postmenopausal KOA through blocking the Wnt/ $\beta$ catenin signaling pathway, providing a promising TCM candidate for KOA therapy. The study is expected to develop a new Chinese medicine formula and dosage form mainly composed of BGRJ, which will have great social and economic benefits after clinical application.

\section{Materials and methods}

\section{Ovariectomized rat model of KOA and BGRJ treatment}

Twenty-five 6-month-old female Sprague-Dawley rats were brought from Qing Long Shan animal breeding grounds (Nanjing, China). Rats were raised in standard conditions with a natural light-dark cycle and were normally fed with free access to water. All rats were randomly divided into five groups $(n=5)$ : Sham group, Model (ovariectomized rat of KOA) group, LPA (glucosamine potassium sulfate capsules) group, M-BGRJ (medium dosage of BGRJ) group and H-BGRJ (high dosage of BGRJ) group. All the rats except those in sham group were performed bilateral ovariectomy (OVX) to induce an ovariectomized rat model of KOA. After 7 weeks, ovariectomized rat of KOA were, respectively, administered with $3.02 \mathrm{~g} / \mathrm{kg}$ and $6.04 \mathrm{~g} / \mathrm{kg}$ BGRJ by gavage in M-BGRJ group and H-BGRJ group, twice each day. In LPA group, ovariectomized rat of KOA were administered with $2.725 \mathrm{mg} / \mathrm{kg}$ glucosamine potassium sulfate capsules solution by gavage, twice each day. Rats in sham group and model group were given $0.9 \%$ normal saline in 
the same volume. During the whole experiment, weights of rats were recorded every 7 days. Three weeks after administration, the rats were intraperitoneally anesthetized with $10 \%$ chloral hydrate solution. The condition of articular cartilage was observed by X-ray. Blood was collected from abdominal aorta, centrifuged at $3000 \mathrm{r}$ for $15 \mathrm{~min}$, and the supernatant was stored in $-80^{\circ} \mathrm{C}$ refrigerator. The distal femur, proximal tibia and medial gastrocnemius of the right lower limb were harvested for further analysis. All experiments were approved by the Animal Care and Use Committee of Nantong TCM Hospital.

\section{Hematoxylin-eosin (H\&E) staining and immunohistochemistry (IHC)}

The articular cartilage was fixed in $10 \%$ formalin solution for $24 \mathrm{~h}$, decalcified in $15 \%$ EDTA-2Na solution at room temperature for 7 days and embedded in paraffin wax. For histological examination, $6-\mu \mathrm{m}$ sections were deparaffinized and rehydrated using graded ethanol $(100 \%, 90 \%$, and $70 \%)$ series and stained with hematoxylin and eosin. For immunohistochemical study, $6 \mu \mathrm{m}$ sections were treated with $10 \% \mathrm{H}_{2} \mathrm{O}_{2}$ for $10 \mathrm{~min}$ at $37{ }^{\circ} \mathrm{C}$, followed by incubation of goat serum for $15 \mathrm{~min}$ at $37^{\circ} \mathrm{C}$. Then, sections were incubated overnight at $4{ }^{\circ} \mathrm{C}$ with the primary antibodies containing Wnt5a, $\beta$-catenin, Sox9 and Collagen II. After PBS washing for three times, sections were incubated with goat anti-rabbit secondary antibody for $30 \mathrm{~min}$ at $37^{\circ} \mathrm{C}$ and incubated with HRP-labeled streptavidin for $15 \mathrm{~min}$ at $37^{\circ} \mathrm{C}$. After washing in PBS for three times, sections were stained with 3, 3 diaminobenzidinetetrahydrochloride (DAB) and nuclei were counterstained with hematoxylin, which were observed under a light microscope.

\section{ELISA assay and nitric oxide (NO) detection}

Serum interleukin-6 (IL-6), interleukin 1- $\beta$ (IL-1 $\beta$ ) and tumor necrosis factor-alpha (TNF- $\alpha$ ) levels were measured using the corresponding rat enzyme-linked immunosorbent assay (ELISA) kits (Beyotime) according to the manufacturer's instructions. Serum NO was determined using NO assay kit (Beyotime).

\section{Preparation of medicine serum}

Eighteen adult Sprague-Dawley female rats were randomly divided into three groups $(n=6)$ : medicine serum 1 group, Medicine serum 2 group and Drug-free ("blank") serum group. Rats in medicine serum 1 and 2 groups were, respectively, administered with $1.51 \mathrm{~g} / \mathrm{kg}$ and $6.04 \mathrm{~g} / \mathrm{kg}$ BGRJ $(0.805 \mathrm{~g} / \mathrm{mL})$ by gavage for consecutive 3 days. On the morning of the 4th day, $10 \mathrm{~mL}$ of blood was collected from the abdominal aorta $1 \mathrm{~h}$ after gavage (fasting and free drinking water $12 \mathrm{~h}$ before blood collection) and centrifuged at $2000 \mathrm{r} / \mathrm{min}$. After centrifugation for $10 \mathrm{~min}$, plasma was collected and stored in the $-20^{\circ} \mathrm{C}$ refrigerator for later use.

\section{Cell culture and cell transfection}

ATDC5 mouse chondroprogenitor cells were purchased from Sigma Aldrich. ATDC5 cells were cultured in Dulbecco's modified Eagle medium (DMEM) and Nutrient F-12 Ham (1:1) containing $5 \%$ fetal bovine serum and $1 \%$ antibiotic-antimycotics at $37{ }^{\circ} \mathrm{C}$ in an atmosphere of $5 \% \mathrm{CO}_{2}$. ATDC5 cells were transfected with siRNA-NC, wnt5a siRNA1, wnt5a siRNA-2 and wnt5a siRNA-3 using Lipofectamine ${ }^{\circledR}$ RNAi MAX Reagent. After 
transfection for $48 \mathrm{~h}$, the transfection effects of cells in each group were detected by RTqPCR. ATDC5 cells in control check (CK) group were normally cultured.

\section{RT-qPCR analysis}

Total RNA in ATDC5 cells was isolated using the RNA easy Mini kit and reverse transcription was performed using the Quanti-Tec ${ }^{\circledR}$ reverse transcription kit for first-strand cDNA synthesis. Then, cDNAs were amplified by PCR assays with SYBR Green Mix kits, and the relative wnt5a expression was standardized with $2^{-\Delta \Delta \mathrm{Ct}}$ method. The PCR program was $95{ }^{\circ} \mathrm{C}$ for $10 \mathrm{~min}$, followed by 44 cycles of denaturation at $95{ }^{\circ} \mathrm{C}$ for $10 \mathrm{~s}$, annealing at $60{ }^{\circ} \mathrm{C}$ for $20 \mathrm{~s}$ and extension at $72{ }^{\circ} \mathrm{C}$ for $10 \mathrm{~s}$. The primers employed were as follows: wnt5a forward, $5^{\prime}$-TTCAACTCCCCGACCACG-3' ${ }^{\prime}$ and reverse, $5^{\prime}$-ATAGCC ACGCCCACAGCA-3'; GAPDH forward, $5^{\prime}$-GGTGAAGGTCGGTGTGAACG-3' ${ }^{\prime}$ and reverse $5^{\prime}$-CTCGCTCCTGGAAGATGGTG-3'.

\section{TUNEL assay}

When ATDC5 cells reached the logarithmic growth stage, the cell concentration was adjusted to $1 \times 10^{5}$ cells $/ \mathrm{mL}$, and the cells were seeded into 48-well plate with $0.3 \mathrm{~mL}$ per well. ATDC5 cells were transfected with siRNA-NC and wnt5a siRNA as above described. siRNA-NC transfected ATDC5 cells were induced by LPS $(5 \mu \mathrm{g} /$ $\mathrm{mL}$ ) and treated with blank serum in siRNA-NC+LPS + blank serum group. wnt5a siRNA transfected ATDC5 cells were induced by LPS $(5 \mu \mathrm{g} / \mathrm{mL})$ and, respectively, treated with blank serum (wnt5a siRNA + LPS + blank serum group), medicine serum 1 (wnt5a siRNA + LPS + medicine serum 1 group) and medicine serum 2 (wnt5a siRNA + LPS + medicine serum 2 group). ATDC5 cells were only treated with blank serum in blank serum group and ATDC5 cells were induced by LPS $(5 \mu \mathrm{g} / \mathrm{mL})$ and treated with blank serum in LPS + blank serum group. After 24-h treatment, TUNEL assay was done on all ATDC5 cells. ATDC5 cells were washed with PBS once and fixed with $4 \%$ paraformaldehyde for $30 \mathrm{~min}$. After PBS washing, ATDC5 cells were incubated in PBS containing $0.3 \%$ Triton X-100 at room temperature for 5 min. $50 \mu \mathrm{L}$ TUNEL working solution was added to each well for incubation at $37^{\circ} \mathrm{C}$ for $1 \mathrm{~h}$ in the dark. After washing with PBS for three times, ATDC5 cells were incubated in DAPI at $37{ }^{\circ} \mathrm{C}$ for $10 \mathrm{~min}$ in the dark. Apoptotic cells (green fluorescence staining) were detected by fluorescence microscope.

\section{Western blot analysis}

Total proteins in ATDC5 cells and cartilage tissues were extracted in RIPA lysis buffer and the lysis extract was centrifuged at $12,000 \mathrm{r} / \mathrm{min}$ for $5 \mathrm{~min}$ at $4{ }^{\circ} \mathrm{C}$. The concentration of total protein supernatant was determined using BCA assay kit. $25 \mu \mathrm{g}$ protein samples were separated with $12 \%$ SDS-PAGE and transferred to a polyvinylidene-difluoride (PVDF) membrane using a wet electroblotting apparatus. Blocked with 5\% skimmed milk powder for $1 \mathrm{~h}$ at room temperature, membranes were probed overnight at $4{ }^{\circ} \mathrm{C}$ with primary antibodies against Wnt5a, $\beta$-catenin, Sox 9 , Collagen II and GAPDH. Next day, membranes were incubated with horseradish peroxidase-linked IgG secondary antibody at 1:5000 dilution for $2 \mathrm{~h}$ at room temperature. Band intensities were obtained using a digital image scanner. 


\section{Statistical analysis}

SPSS version 22.0 was used for the analysis of data which were presented as mean \pm standard deviation (SD). The significance of differences between multiple groups was evaluated by one-way analysis of variance (ANOVA) followed by post hoc Tukey's test. $P<0.05$ was considered statistically significant.

\section{Abbreviations}

KOA: Knee osteoarthritis; BGRJ: Bugan Rongjin decoction; IL-6: Interleukin-6; TNF-a: Tumor necrosis factor-alpha; NO: Nitric oxide; H\&E: Hematoxylin-eosin; IHC: Immunohistochemistry; PF: Paeoniflorin; I/R: Ischemia/reperfusion; LPS: Lipopolysaccharide; HA: Hyaluronic acid; ECM: Extracellular matrix; OVX: Ovariectomy; ELISA: Enzyme-linked immunosorbent assay; DMEM: Dulbecco's modified Eagle medium; CK: Control check; PVDF: Polyvinylidene difluoride; SD: Standard deviation; ANOVA: Analysis of variance.

\section{Acknowledgements}

Not applicable.

\section{Authors' contributions}

SW: project development, data analysis and collection, manuscript writing. PD: project development, data analysis and collection, manuscript writing. XX: project development, data collection. XC: project development, data collection. DM: project development, data collection. SS: project development, data analysis and collection. FG: project development, data collection. ZL: project development, data analysis and collection. KS: project development, manuscript editing. YL: project development, manuscript editing. All authors read and approved the final manuscript.

\section{Funding}

Command project of Nantong Science and Technology Bureau (MS12019036); Nantong Health Commission Youth Fund Project (QA2019016).

\section{Availability of data and materials}

The experimental data will be available on the request.

\section{Declarations}

\section{Ethics approval and consent to participate}

All experiments were approved by the Animal Care and Use Committee of Nantong TCM Hospital.

\section{Consent for publication}

All authors have read the manuscript and approved for publication.

\section{Competing interests}

The authors declare they have no competing interests.

\section{Author details}

${ }^{1}$ Department of Orthopedics and Traumatology, Nantong TCM Hospital Affiliated to Nanjing University of Chinese Medicine, Nantong 226001, Jiangsu, China. ${ }^{2}$ Department of Pediatrics, Nantong TCM Hospital Affiliated to Nanjing University of Chinese Medicine, Nantong 226001, Jiangsu, China. ${ }^{3}$ Department of Science and Education, Nantong TCM Hospital Affiliated to Nanjing University of Chinese Medicine, Nantong 226001, Jiangsu, China. ${ }^{4}$ Department of Respiratory Medicine, Affiliated Hospital of Integrated Traditional Chinese and Western Medicine, Nanjing University of Chinese Medicine, Number 100, Cross Street, Hongshan Road, Qixia District, Nanjing 210028, Jiangsu, China. ${ }^{5}$ Department of Orthopedics and Traumatology, Suzhou TCM Hospital Affiliated to Nanjing University of Chinese Medicine, Number 18 Yangsu Road, Suzhou 215000, Jiangsu, China.

Received: 17 June 2021 Accepted: 25 September 2021

Published online: 13 October 2021

\section{References}

1. Berenbaum F. Osteoarthritis as an inflammatory disease (osteoarthritis is not osteoarthrosis!). Osteoarthr Cartil. 2013;21(1):16-21.

2. Johnson VL, Hunter DJ. The epidemiology of osteoarthritis. Best Pract Res Clin Rheumatol. 2014;28(1):5-15.

3. Glyn-Jones S, Palmer AJ, Agricola R, Price AJ, Vincent TL, Weinans H, Carr AJ. Osteoarthritis. Lancet. 2015:386(9991):376-87.

4. Liao D. Epidemiological investigation of osteoarthritis in China. J Minimally Invasive Med. 2017;12(4):521-4.

5. Boyan BD, Hart DA, Enoka RM, Nicolella DP, Resnick E, Berkley KJ, Sluka KA, Kwoh CK, Tosi LL, O'Connor MI, et al. Hormonal modulation of connective tissue homeostasis and sex differences in risk for osteoarthritis of the knee. Biol Sex Differ. 2013:4(1):3-13.

6. Guo J, Chen Y, Li Z, Cheng S, Tang C, Dong X, He W, Huang Y, Yin B, Sheng Y, et al. The cerebral mechanism of acupuncture for treating knee osteoarthritis: study protocol for a randomized controlled trial. Trials. 2019;20(1):126. 
7. Wang H, Liu J, Zhao Y. Progress on integrated Chinese and Western medicine in the treatment of osteoarthritis. Chin J Integr Med. 2010;16(4):378-84.

8. Qin Y. Study on syndrome differentiation of traditional Chinese medicine meridian tendons in knee joint osteoarthritis. Doctor. Beijing University of Chinese Medicine; 2010.

9. Liu W. Therapeutic effects comparison between acupuncture combined with Bugu Jianxi recipe and simple acupuncture for knee osteoarthritis and the impact on relevant indexes. J Sichuan Trad Chin Med. 2017;35(6):122-5.

10. Zeng L, Yang W, Pan J, Guo D, Luo M, Liang H, Lin J, Han Y, Fang J, Ou A, et al. Adjuvant treatment by TCM prescription of Shenyu Hezhi approach in knee osteoarthritis: a systematic review of literature. China J Trad Chin Med Pharm. 2017;32(11):5088-95.

11. Meng Q, Zhou Z, Gu H, Du X, Zheng F, Guo H. The clinical application experience of Zhu Liangchun Chinese medical master in treating arthralgia. Liaoning JTrad Chin Med. 2012;39(05):791-2.

12. Xie T, Li K, Gong X, Jiang R, Huang W, Chen X, Tie H, Zhou Q, Wu S, Wan J, Wang B. Paeoniflorin protects against liver ischemia/reperfusion injury in mice via inhibiting HMGB1-TLR4 signaling pathway. Phytother Res PTR. 2018;32(11):2247-55.

13. Zeng YF, Wang R, Bian Y, Chen WS, Peng L. Catalpol attenuates IL-1 $\beta$ induced matrix catabolism, apoptosis and inflammation in rat chondrocytes and inhibits cartilage degeneration. Med Sci Monit. 2019;25:6649-59.

14. Wang F, Wu L, Li L, Chen S. Monotropein exerts protective effects against IL-1 $\beta$-induced apoptosis and catabolic responses on osteoarthritis chondrocytes. Int Immunopharmacol. 2014;23(2):575-80.

15. Zhuang $C$, Wang $Y$, Zhang $Y, X u$ N. Oxidative stress in osteoarthritis and antioxidant effect of polysaccharide from angelica sinensis. Int J Biol Macromol. 2018;115:281-6.

16. Zhang S, Zhang Q, Zhang D, Wang C, Yan C. Anti-osteoporosis activity of a novel Achyranthes bidentata polysaccharide via stimulating bone formation. Carbohyd Polym. 2018;184:288-98.

17. He G, Guo W, Lou Z, Zhang H. Achyranthes bidentata saponins promote osteogenic differentiation of bone marrow stromal cells through the ERK MAPK signaling pathway. Cell Biochem Biophys. 2014;70(1):467-73.

18. Ju L, Hu P, Chen P, Xue X, Li Z, He F, Qiu Z, Cheng J, Huang F. Huoxuezhitong capsule ameliorates MIA-induced osteoarthritis of rats through suppressing PI3K/Akt/NF-KB pathway. Biomed Pharmacother. 2020;129:110471.

19. Liu L, Wang LP, He S, Ma Y. Immune homeostasis: effects of Chinese herbal formulae and herb-derived compounds on allergic asthma in different experimental models. Chin J Integr Med. 2018;24(5):390-8.

20. Lv S, Junjie Z, Xie X, Gao W, Chen X, Liu C. The clinical use and its mechanisms research on angelicae pubescentis and loranthi decoction in KOA treatment. Global Trad Chin Med. 2015;8(09):1149-52.

21. Kim KS, Cho HS, Lee SD, Kim KH, Cho JY, Chung KH, Lee YC, Moon SK, Kim CH. Inhibitory effect of Buthus martensi Karsch extracts on interleukin-1 beta-induced expression of nitric oxide (NO) synthase and production of NO in human chondrocytes and LPS-induced NO and prostaglandin E2 production in mouse peritoneal macrophages. Toxicol In Vitro Int J Publ Assoc BIBRA. 2005;19(6):757-69.

22. Dong R, Tan Y, Fan A, Liao Z, Liu H, Wei P. Molecular dynamics of the recruitment of immunoreceptor signaling module DAP12 homodimer to lipid raft boundary regulated by PIP2. J Phys Chem B. 2020;124(3):504-10.

23. Philp AM, Davis ET, Jones SW. Developing anti-inflammatory therapeutics for patients with osteoarthritis. Rheumatology (Oxford). 2017;56(6):869-81

24. Li $H$, Peng $Y$, Wang $X$, Sun $X$, Yang F, Sun $Y$, Wang B. Astragaloside inhibits IL-1 $\beta$-induced inflammatory response in human osteoarthritis chondrocytes and ameliorates the progression of osteoarthritis in mice. Immunopharmacol Immunotoxicol. 2019;41(4):497-503.

25. Gao J, Liu X. Effects of electroacupuncture combined with Weilingxian intra-articular injection on TNF-a, MMPs and HA in rats with knee osteoarthritis. Chin J Immun. 2019;35(06):686-92.

26. Lou F, Yuan S, Jia H, Li X, Dong S. Effect of gutong powder on the expression of IL-1 $\beta$, TNF-a and MMP-3 in the patients with knee osteoarthritis. Clin J Trad Chin Med. 2017;29(11):1912-5.

27. Mohammed MK, Shao C, Wang J, Wei Q, Wang X, Collier Z, Tang S, Liu H, Zhang F, Huang J, et al. Wnt/ $\beta$-catenin signaling plays an ever-expanding role in stem cell self-renewal, tumorigenesis and cancer chemoresistance. Genes Dis. 2016:3(1):11-40

28. Takahito Y, Tomohiro O, Tatsuya K, Masahiro I, Motomi E-I. Wnt/beta-catenin signaling stimulates matrix catabolic genes and activity in articular chondrocytes: its possible role in joint degeneration. Lab Invest J Tech Methods Pathol. 2008;88(3):264-74.

29. Dubin A. Managing osteoarthritis and other chronic musculoskeletal pain disorders. Med Clin North Am. 2016;100(1):143-50

30. Usami Y, Gunawardena AT, Iwamoto M, Enomoto-Iwamoto M. Wnt signaling in cartilage development and diseases: lessons from animal studies. Lab Invest. 2016;96(2):186-96.

31. Tang M, Ni H, Zhang X, Shao L. Clinical study on the role of reinforcing kidney activating blood drugs (TCD) in the regulation of classic Wnt/ $\beta$-catenin pathway in patients with osteoarthritis. J Trad Chin Orthopedics Traumatol. 2014;26(08):12-1417.

32. Ning X, Liu H, Zhang J, Zhu H. Study on histology of herbs invigorating kidney cure knee osteoarthritis. J Clin Med. 2014;1(12):2080-1.

\section{Publisher's Note}

Springer Nature remains neutral with regard to jurisdictional claims in published maps and institutional affiliations. 